\title{
Self-consistent Analysis of Doping Effect for Magnetic Ordering in Stacked-Kagome Weyl System
}

\author{
Akihiro Ozawa周 and Kentaro Nomura ${ }^{1,2}$ \\ ${ }^{1}$ Institute for Materials Research, Tohoku University, Katahira, Aoba-ku, Sendai 980-8577 and \\ ${ }^{2}$ Center for Spintronics Research Network, Tohoku University, Katahira, Aoba-ku, Sendai 980-8577
}

\begin{abstract}
We theoretically study the carrier doping effect for magnetism in a stacked-kagome system $\mathrm{Co}_{3} \mathrm{Sn}_{2} \mathrm{~S}_{2}$ based on an effective model and the Hartree-Fock method. We show the electron filling and temperature dependences of the magnetic order parameter. The perpendicular ferromagnetic ordering is suppressed by hole doping, wheres undoped $\mathrm{Co}_{3} \mathrm{Sn}_{2} \mathrm{~S}_{2}$ shows magnetic Weyl semimetal state. Additionally, in the electron-doped regime, we find a non-collinear antiferromagnetic ordering. Especially, in the non-collinear antiferromagnetic state, by considering a certain spin-orbit coupling, the finite orbital magnetization and the anomalous Hall conductivity are obtained.
\end{abstract}

\section{INTRODUCTION}

Magnetic kagome-lattice systems such as $\mathrm{Mn}_{3} \mathrm{Sn}[1]$, $\mathrm{Fe}_{3} \mathrm{Sn}_{2}$ [6, 7], and $\mathrm{Co}_{3} \mathrm{Sn}_{2} \mathrm{~S}_{2}$ [8 12 (CSS) are attracting a great deal of attentions because of their diverse electronic and magnetic properties. The anomalous Hall effect, originated from the topological gapless points in momentum space called the Weyl points 13 15, is one of the significant transport properties in these materials. Especially, CSS possesses the small Fermi surface with the Weyl points and is called the Weyl semimetal[8]. In addition to the electronic properties, these systems show different magnetic ordering, although they commonly have kagome-lattice layers [16. $\mathrm{Mn}_{3} \mathrm{Sn}$ shows a noncollinear antiferromagnetic (AF) arrangement in which the magnetic moments of $\mathrm{Mn}$ are oriented at a relative angle of $120^{\circ}$ in the kagome plane 1]. $\mathrm{Fe}_{3} \mathrm{Sn}_{2}$ shows ferromagnetic $(\mathrm{FM})$ ordering with the in-plane magnetic anisotropy [6, 7]. In CSS, although the ground state shows perpendicular FM ordering [8, 17, 18, recent experiments predict a non-collinear AF arrangement at finite temperature 19 21. According to the theory of metallic magnetism [22], it has been established that the Fermi surface structure plays an important role for magnetic ordering. Therefore, it is expected that the magnetic ordering is altered by tuning the Fermi level. However, the theoretical investigations for the magnetic ordering with different Fermi levels in stacked-kagome systems are not well achieved.

In this paper, based on the effective model of the magnetic Weyl semimetal CSS 23, we study the magnetic ordering with respect to the experimentally controllable parameters, the filling factor of dopants and temperature. Our results for magnetic ordering are summarized as a schematic picture in Fig. 1. A non-collinear AF ordering appears by electron doping, wheres undoped system shows the perpendicular ferromagnetic Weyl state. As characteristic properties in the non-collinear AF state,

\footnotetext{
* akihiro.ozawa.s4@dc.tohoku.ac.jp

† kentaro.nomura.e7@tohoku.ac.jp
}

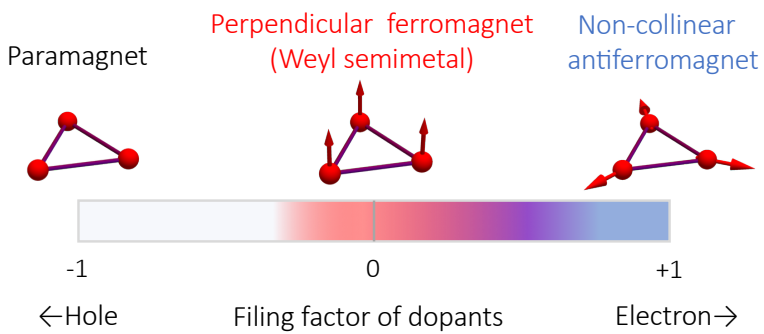

FIG. 1. Possible phases in doped $\mathrm{Co}_{3} \mathrm{Sn}_{2} \mathrm{~S}_{2}$. In undoped $\mathrm{Co}_{3} \mathrm{Sn}_{2} \mathrm{~S}_{2}$, Weyl semimetal phase with perpendicular ferromagnetic ordering appears. In hole-doped $\mathrm{Co}_{3} \mathrm{Sn}_{2} \mathrm{~S}_{2}$, the ferromagnetic ordering is suppressed and the system becomes paramagnetic. In electron-doped $\mathrm{Co}_{3} \mathrm{Sn}_{2} \mathrm{~S}_{2}$, a non-collinear antiferromagnetic ordering appears.

the orbital magnetization and the anomalous Hall conductivity become finite by considering a certain spinorbit coupling.

\section{TIGHT-BINDING HAMILTONIAN AND HARTREE-FOCK MEAN-FIELD FORMALISM}

First, we briefly introduce the effective model of CSS. In our previous study[23, we constructed an effective two-orbital model of CSS, by considering few orbitals. This model reproduces the electronic band structure which is similar to that obtained by first-principles calculations 8, 9. Figure 2(a) shows the original crystal structure of CSS. The stackcked kagome layers consist of Co and sandwich two types of triangle layers which consist of Sn and S, respectively. In the effective model, one $d$ orbital from Co forming kagome layers and $p$ orbital from interlayer Sn are extracted as a dashed box in Fig. 2(a) shows. All other orbitals are neglected in the following for simplicity. The primitive translation vectors are $\boldsymbol{a}_{1}=\left(\frac{a}{2}, 0, c\right), \boldsymbol{a}_{2}=\left(-\frac{a}{4}, \frac{\sqrt{3} a}{4}, c\right), \boldsymbol{a}_{3}=\left(-\frac{a}{4},-\frac{\sqrt{3} a}{4}, c\right)$. In the following we set $c=\frac{\sqrt{3} a}{2}$ for simplicity. The hopping 
term of this model is given by,

$$
H_{0}=H_{\mathrm{d}-\mathrm{p}}+H_{\mathrm{KM}} .
$$

$H_{\mathrm{d}-\mathrm{p}}$ is the spin independent hopping term, $H_{\mathrm{KM}}$ is the spin-orbit coupling term. First, we explain $H_{\mathrm{d}-\mathrm{p}}$,

$$
\begin{array}{r}
H_{\mathrm{d}-\mathrm{p}}=-\sum_{i j \sigma}\left[t_{i j} d_{i \sigma}^{\dagger} d_{j \sigma}+t_{i j}^{\mathrm{dp}}\left(d_{i \sigma}^{\dagger} p_{j \sigma}+p_{i \sigma}^{\dagger} d_{j \sigma}\right)\right] \\
+\epsilon_{\mathrm{p}} \sum_{i \sigma} p_{i \sigma}^{\dagger} p_{i \sigma} .
\end{array}
$$

$d_{i \sigma}$ and $p_{i \sigma}$ are the annihilation operators of $d$ orbital on the kagome lattice and $p$ orbital on the triangle lattice, respectively. $t_{i j}$ includes the first and second-nearest neighbor hopping, $t_{1}$ and $t_{2}$, in the intra-kagome layer, inter-kagome layer hopping $t_{z} \cdot t^{\mathrm{dp}}$ indicates $d p$ hybridization between $d$ orbital of Co and $p$ orbital of Sn. $\epsilon_{p}$ is the on-site potential of $p$ orbital on Sn. $H_{\mathrm{KM}}$ describes the Kane-Mele type SOC term [24, 25] on the intra kagome layer given as follows,

$$
H_{\mathrm{KM}}=-\mathrm{i} t_{\mathrm{KM}} \sum_{\langle\langle i j\rangle\rangle \sigma \sigma^{\prime}} \nu_{i j} \cdot d_{i \sigma}^{\dagger} \sigma_{\sigma \sigma^{\prime}}^{z} d_{j \sigma^{\prime}} .
$$

$t_{\mathrm{KM}}$ is the hopping strength and the summation $\langle\langle i j\rangle\rangle$ is about intra layer second-nearest-neighbor sites. The sign is $\nu_{i j}=+1(-1)$, when the electron moves counterclockwise (clockwise) to get to the second-nearest-neighbor site on the kagome plane 24, 25]. Spin-orbit coupling plays a role to obtain the Weyl points [8, 23].

Next, we construct the mean-field Hamiltonian by using the Hartree-Fock approximation. In order to discuss the itinerant magnetism due to the electron correlation, we introduce the on-site Coulomb interaction term. The on-site Coulomb interaction terms for $d$ orbital $H_{d d}^{\mathrm{U}}$ and $p$ orbital $H_{p p}^{\mathrm{U}}$ are respectively given by,

$$
\begin{gathered}
H_{d d}^{\mathrm{U}}=U_{d d} \sum_{i} \sum_{\alpha} d_{i \alpha \uparrow}^{\dagger} d_{i \alpha \downarrow}^{\dagger} d_{i \alpha \downarrow} d_{i \alpha \uparrow}, \\
H_{p p}^{\mathrm{U}}=U_{p p} \sum_{i} p_{i \uparrow}^{\dagger} p_{i \downarrow}^{\dagger} p_{i \downarrow} p_{i \uparrow} .
\end{gathered}
$$

$U_{d d}$ and $U_{p p}$ are the bare on-site Coulomb interaction strengths of $d$ orbital on Co and of $p$ orbital on Sn, respectively. $i$ and $\alpha=\mathrm{A}, \mathrm{B}$, or $\mathrm{C}$ indicate the position of the unit cell and the sublattice index of Co, respectively. We assume that the fluctuation of the magnetic moment is small. Thus we introduce the Hartree-Fock approximation $H_{d d}^{U} \sim H_{d d}^{H F}, H_{p p}^{U} \sim H_{p p}^{H F}$ for the twobody operators in Eq. (4) and Eq. (5) as,

$$
\begin{gathered}
H_{d d}^{\mathrm{HF}}=U_{d d} \sum_{i \alpha}\left[\left\langle n_{i \alpha \uparrow}\right\rangle n_{i \alpha \downarrow}+\left\langle n_{i \alpha \downarrow}\right\rangle n_{i \alpha \uparrow}-\left\langle n_{i \alpha \uparrow}\right\rangle\left\langle n_{i \alpha \downarrow}\right\rangle\right. \\
-\left\langle d_{i \alpha \uparrow}^{\dagger} d_{i \alpha \downarrow}\right\rangle d_{i \alpha \downarrow}^{\dagger} d_{i \alpha \uparrow}-\left\langle d_{i \alpha \downarrow}^{\dagger} d_{i \alpha \uparrow}\right\rangle d_{i \alpha \uparrow}^{\dagger} d_{i \alpha \downarrow} \\
\left.+\left\langle d_{i \alpha \uparrow}^{\dagger} d_{i \alpha \downarrow}\right\rangle\left\langle d_{i \alpha \downarrow}^{\dagger} d_{i \alpha \uparrow}\right\rangle\right]
\end{gathered}
$$

$$
H_{p p}^{\mathrm{HF}}=U_{p p} \sum_{i}\left[\left\langle n_{i p \uparrow}\right\rangle n_{i p \downarrow}+\left\langle n_{i p \downarrow}\right\rangle n_{i p \uparrow}-\left\langle n_{i p \uparrow}\right\rangle\left\langle n_{i p \downarrow}\right\rangle\right] .
$$

$n_{i \alpha \sigma}=d_{i \alpha \sigma}^{\dagger} d_{i \alpha \sigma}$ and $n_{i p \sigma}=p_{i \sigma}^{\dagger} p_{i \sigma}$ are the particle number operators of Co and Sn, with spin $\sigma$ on $i$ th unit cell, respectively. We neglect the in-plane component of magnetization on Sn site for simplicity. The total mean-field Hamiltonian $H_{\mathrm{MF}}$ is given by,

$$
H_{\mathrm{MF}}=H_{0}+H_{d d}^{\mathrm{HF}}+H_{p p}^{\mathrm{HF}} .
$$

We assume that the translational symmetry of the crystal structure remains even in the magnetically ordered phase. The mean-field Hamiltonian in momentum space can be obtained by using the Fourier transformation $d_{i \alpha \sigma}=\frac{1}{\sqrt{N}} \sum_{\boldsymbol{k}} \mathrm{e}^{i \boldsymbol{k} \cdot \boldsymbol{R}_{i}} d_{\boldsymbol{k} \alpha \sigma}, \quad p_{i \sigma}=\frac{1}{\sqrt{N}} \sum_{\boldsymbol{k}} \mathrm{e}^{i \boldsymbol{k} \cdot \boldsymbol{R}_{i}} p_{\boldsymbol{k} \sigma}$. Here $\boldsymbol{k}$ is the crystal momentum and $N$ is the number of unit cells. The Bloch Hamiltonian matrix $\mathcal{H}_{\mathrm{MF}}(\boldsymbol{k})$ can be written in the form, $H_{\mathrm{MF}}=\sum_{\boldsymbol{k}, \sigma} C_{\boldsymbol{k} \sigma}^{\dagger} \mathcal{H}_{\mathrm{MF}}(\boldsymbol{k}) C_{\boldsymbol{k} \sigma}$, where $C_{\boldsymbol{k} \sigma}^{\dagger}=\left(d_{\boldsymbol{k} A \sigma}^{\dagger}, d_{\boldsymbol{k} B \sigma}^{\dagger}, d_{\boldsymbol{k} C \sigma}^{\dagger}, p_{\boldsymbol{k} \sigma}^{\dagger}\right)$ and $\mathcal{H}_{\mathrm{MF}}(\boldsymbol{k})$ is given by $8 \times 8$ matrix,

$$
\mathcal{H}_{\mathrm{MF}}(\boldsymbol{k})=\mathcal{H}_{0}(\boldsymbol{k})+\mathcal{H}_{\mathrm{exc}}+H_{E},
$$

in momentum space. $\mathcal{H}_{\text {exc }}$ is the exchange term which describes coupling between the mean-field parameter and spins of electrons as,

$$
\mathcal{H}_{\mathrm{exc}}=-\frac{U}{2} \operatorname{diag}\left[\boldsymbol{\sigma} \cdot\left\langle\boldsymbol{m}_{A}\right\rangle, \boldsymbol{\sigma} \cdot\left\langle\boldsymbol{m}_{B}\right\rangle, \boldsymbol{\sigma} \cdot\left\langle\boldsymbol{m}_{C}\right\rangle, \sigma_{z}\left\langle m_{S}^{z}\right\rangle\right] .
$$

$\boldsymbol{\sigma}$ is the vector of Pauli matrices which corresponds to the spin of electron. $\left\langle\boldsymbol{m}_{\alpha}\right\rangle$ and $\left\langle m_{S}^{z}\right\rangle$ are the meanfield parameters on the $\alpha$ sublattice of Co and Sn, respectively. In this mean-field Hamiltonian Eq. (9), the $z$-component of magnetization and particle number on each site are computed as $\left\langle m_{\gamma}^{z}\right\rangle=\left\langle n_{\gamma \uparrow}\right\rangle-\left\langle n_{\gamma \downarrow}\right\rangle,\left\langle n_{\gamma}\right\rangle=$ $\left\langle n_{\gamma \uparrow}\right\rangle+\left\langle n_{\gamma \downarrow}\right\rangle$. Here, we use the simplified sublattice index as $\gamma \in \alpha, S$, and $\left\langle n_{\gamma \sigma}\right\rangle=\frac{1}{N} \sum_{\lambda, \boldsymbol{k}}\left\langle\lambda, \boldsymbol{k}\left|P_{\sigma}^{\gamma}\right| \lambda, \boldsymbol{k}\right\rangle f\left(E_{\lambda \boldsymbol{k}}-\right.$ $\mu)$. In-plane components can be obtained as, $\left\langle m_{\alpha}^{x}\right\rangle=$ $2 \operatorname{Re}\left\langle d_{\alpha \uparrow}^{\dagger} d_{\alpha \downarrow}\right\rangle,\left\langle m_{\alpha}^{y}\right\rangle=2 \operatorname{Im}\left\langle d_{\alpha \uparrow}^{\dagger} d_{\alpha \downarrow}\right\rangle$, where $\left\langle d_{\alpha \uparrow}^{\dagger} d_{\alpha \downarrow}\right\rangle=$ $\frac{1}{N} \sum_{\lambda, \boldsymbol{k}}\left\langle\lambda, \boldsymbol{k}\left|P^{\alpha} \sigma^{+}\right| \lambda, \boldsymbol{k}\right\rangle f\left(E_{\lambda \boldsymbol{k}}-\mu\right) . f\left(E_{\lambda \boldsymbol{k}}\right)$ is the FermiDirac distribution function. $\mu$ is the chemical potential and discussed in detail in the next section. $P^{\gamma}$ is the projection operators for $\gamma$ site with spin $\sigma . \sigma^{+}$is given by $\sigma^{+}=\sigma_{x}+i \sigma_{y}$. Third term $H_{E}$ is given by,

$$
\begin{aligned}
& H_{E}=\frac{U_{d d}}{4} \operatorname{diag}\left[E_{A}, E_{B}, E_{C}, 0\right]+\frac{U_{p p}}{4} \operatorname{diag}\left[0,0,0, E_{S}\right] \\
& +\frac{U_{d d}}{2} \operatorname{diag}\left[\left\langle n_{A}\right\rangle,\left\langle n_{B}\right\rangle,\left\langle n_{C}\right\rangle, 0\right]+\frac{U_{p p}}{2} \operatorname{diag}\left[0,0,0,\left\langle n_{S}\right\rangle\right]
\end{aligned}
$$

$E_{\alpha}=\left\langle\boldsymbol{m}_{\alpha}\right\rangle^{2}-\left\langle n_{\alpha}\right\rangle^{2}$ and $E_{S}=\left\langle m_{S}^{z}\right\rangle^{2}-\left\langle n_{S}\right\rangle^{2}$. For each $\boldsymbol{k}$, the Bloch state is given as an eight component vector $|\lambda, \boldsymbol{k}\rangle$, where $\lambda$ is the band index. $E_{\lambda \boldsymbol{k}}$ is the eigenvalue of $|\lambda, \boldsymbol{k}\rangle$. The eigenvector $|\lambda, \boldsymbol{k}\rangle$ and order parameters 
(a)

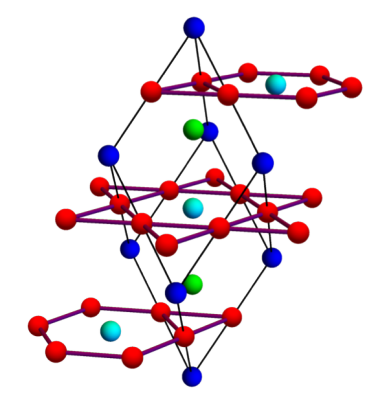

(b) $\Delta n_{\mathrm{e}}=0\left(n_{\mathrm{e}}=3\right) \quad$ cf. $\mathrm{Co}_{3} \mathrm{Sn}_{2} \mathrm{~S}_{2}$

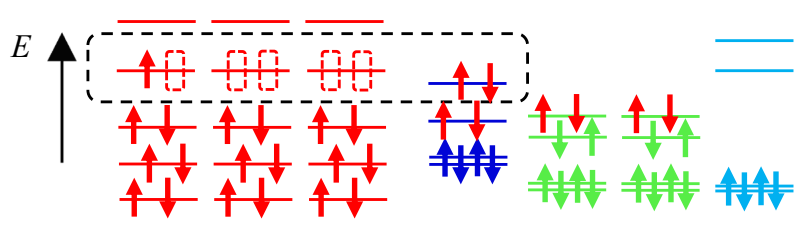

Magnetic Weyl semimetal

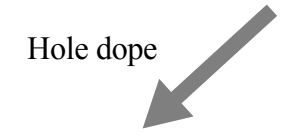

(c) $\Delta n_{\mathrm{e}}=-1\left(n_{\mathrm{e}}=2\right)$ cf. $\mathrm{Co}_{2} \mathrm{FeSn}_{2} \mathrm{~S}_{2}$

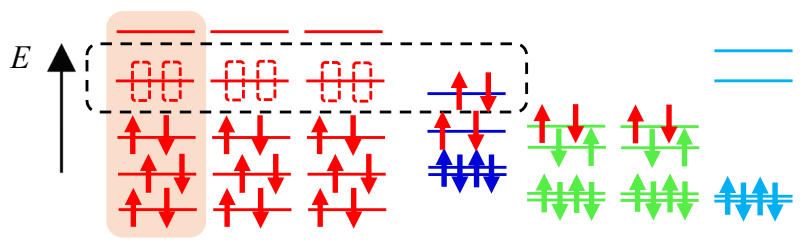

$\mathrm{Fe}$

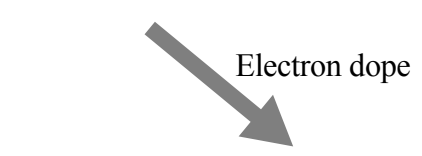

(d) $\Delta n_{\mathrm{e}}=+1\left(n_{\mathrm{e}}=4\right) \quad$ cf. $\mathrm{Co}_{2} \mathrm{NiSn}_{2} \mathrm{~S}_{2}$

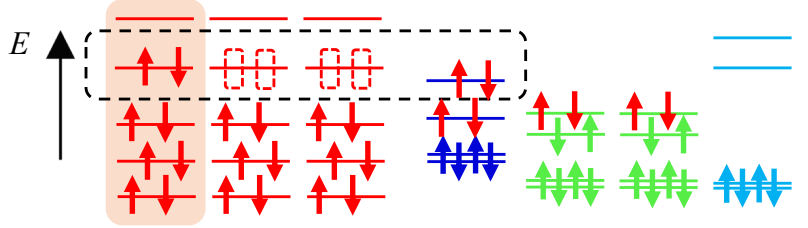

$\mathrm{Ni}$

FIG. 2. (a) Crystal structure of $\mathrm{Co}_{3} \mathrm{Sn}_{2} \mathrm{~S}_{2}$. Co forms the kagome lattice network and sandwiches two layers of triangle lattice formed by $\mathrm{Sn}$ and S, respectively. (b) Anticipated electronic structures of undoped $\mathrm{Co}_{3} \mathrm{Sn}_{2} \mathrm{~S}_{2}$. A dashed box indicates the limited orbitals in our effective model. The total electron number per unit cell is $n_{\mathrm{e}}=3$. (c) Electronic structure when the total electron number is $n_{\mathrm{e}}=2$. In experimental situation, one hole is doped by substituting Co with Fe, in each unit cell. (d) Electronic structure when the total electron number per unit cell is $n_{\mathrm{e}}=4$. In experimental situation, one electron is doped by substituting $\mathrm{Co}$ with $\mathrm{Ni}$, in each unit cell.

$\left\langle\boldsymbol{m}_{\alpha}\right\rangle$ can be obtained by diagonalizing $\mathcal{H}_{\mathrm{MF}}(\boldsymbol{k})$ so that the Eq. (9) should be calculated self-consistently. In the following, we set $t_{1}$ as a unit of energy, $t_{2}=0.6 t_{1}, t_{\mathrm{dp}}=$ $2.35 t_{1}, t_{\mathrm{z}}=-1.2 t_{1}, \epsilon_{p}=-8.5 t_{1}, t_{\mathrm{KM}}=0.2 t_{1}, U_{d d}=$ $7.0 t_{1}$, and $U_{p p}=5.5 t_{1}$. These parameters are chosen to fit the band structure to the result obtained by firstprinciples calculations [8, 26, 27.

\section{CONDITION OF TOTAL NUMBER OF ELECTRONS IN UNIT CELL}

Next, we discuss the chemical potential in our theoretical model. In the following, we assume that the doping effect is considered as only a change of the number of electron per unit cell, and the randomness due to the impurities is neglected. As mentioned in the previous section, we extracted one orbital from five $d$ orbitals of each Co and one orbital from $p$ orbitals of interlayer Sn, and neglected all other orbitals as shown in Fig. 2(a). Therefore, the unit cell has $(3+1) \times 2=8$ states including the spin degrees of freedom in our model. To determine $\mu$ appropriately, we discuss the electronic orbital configurations in the doped CSS. As discussed in our previous paper 23, in the undoped CSS, we assume that one of three sites of Co is occupied by one electron, and interlayer Sn site is occupied by two electrons. Thus the total number of electrons in limited orbitals, is $n_{\mathrm{e}}=3$ per unit cell as shown in Fig. 2(a). This configuration is consistent with the magnetization per unit cell $m_{z} \sim 1.0$ as obtained by experiment $[8$. In this work, we study the doping effect to the undoped CSS. To clearly characterize the filling factor of dopants, we use $\Delta n_{\mathrm{e}}$ as the deviation from $n_{\mathrm{e}}=3$ in the following results. Therefore, $n_{\mathrm{e}}=3$ is equivalent to $\Delta n_{\mathrm{e}}=0$. When one Co in each unit cell is substituted with one Fe, the anticipated electronic orbital configuration is shown in Fig. 2(c). In this case, the total number of electrons per unit cell is $n_{\mathrm{e}}=2$ so $\Delta n_{\mathrm{e}}=-1$. Presumably, even if $\mathrm{Sn}$ is substituted with In, instead of substituting Co with Fe, the total number of electrons per unit cell is same as that in Fig. 2(c). This is because one electron at the Co orbital is expected to move to the In orbital, which is assumed to be energetically low. On the other hand, when one of Co site in each unit cell is substituted with one $\mathrm{Ni}$, the anticipated electronic orbital configuration is shown in Fig. 2(d). In this case, the total number of electrons per unit cell is $n_{\mathrm{e}}=4$ so $\Delta n_{\mathrm{e}}=+1$. The chemical potential $\mu$ is numerically 
(a)

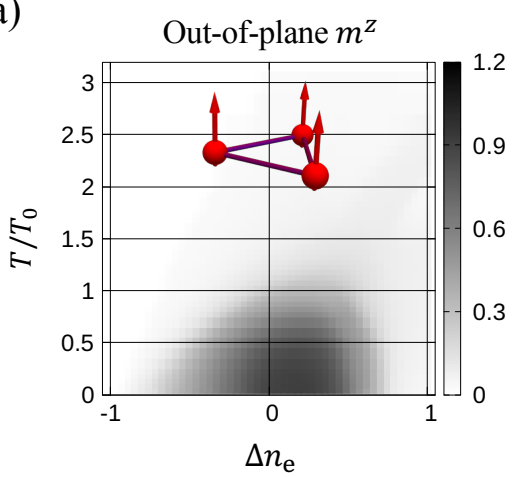

(d)

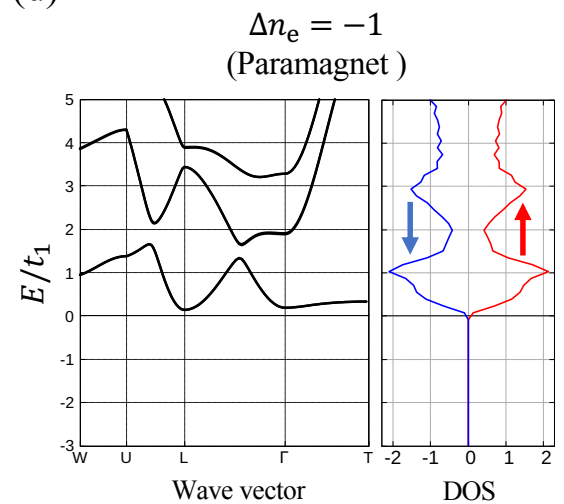

(b)

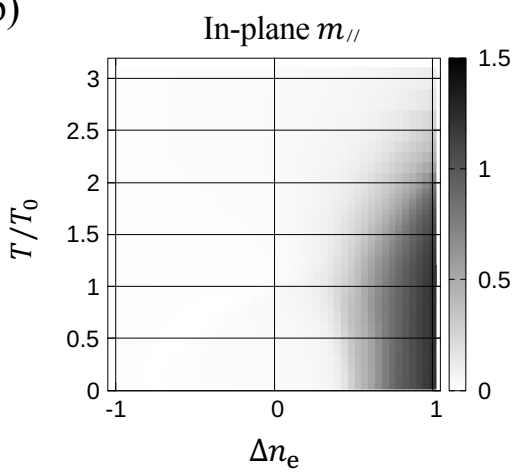

(e)

$$
\Delta n_{\mathrm{e}}=0
$$

(Perpendicular ferromagnet)

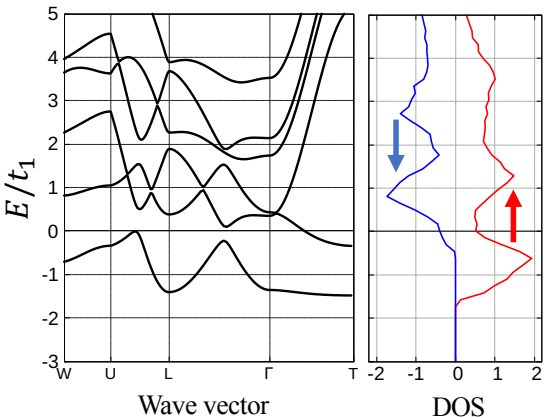

(c)

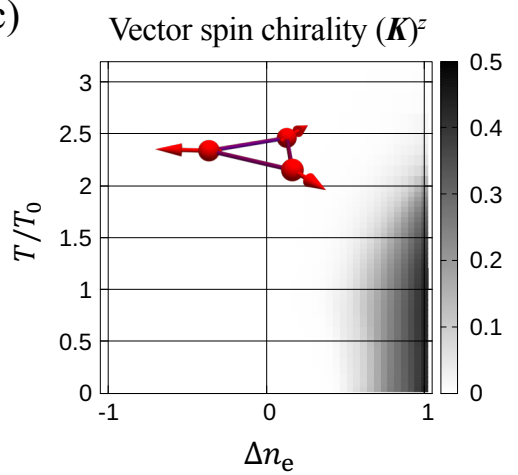

(f)

$$
\Delta n_{\mathrm{e}}=+1
$$

(Non-collinear antiferromagnet)

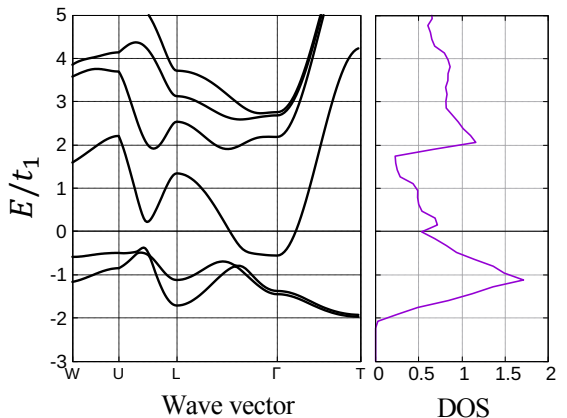

FIG. 3. Color maps for (a) $z$ component of magnetization $m^{z}$ per unit cell in units of $\mu_{\mathrm{B}}$, (b) in-plane component of magnetization and (c) $z$ component of vector spin chirality with respect to the filling factor of dopants and temperature. In case $\Delta n_{\mathrm{e}} \sim 0.0$, (a) ferromagnetic ordering with $m^{z} \sim 0.9$ appears. $m^{z}$ decreases as $\Delta n_{\mathrm{e}}$ deviates from $\Delta n_{\mathrm{e}} \sim 0$. When $\Delta n_{\mathrm{e}} \sim+1.0$, (a) $z$ component of magnetization diminishes, while (b) in-plane component of magnetization and (c) $z$ component of the vector spin chirality become finite, indicating non-collinear antiferromagnetic state. Electronic band structures of (d) paramagnetic state, (e) perpendicular ferromagnetic state and (f) non-collinear antiferromagnetic state obtained by the Hartree-Fock method. In (d) $\Delta n_{\mathrm{e}}=-1$, system is paramagnetic and the chemical potential is close to the band gap. In (e) $\Delta n_{\mathrm{e}}=-1$, system is ferromagnetic, the chemical potential is located near the local minimum of the spin majority band, corresponding to the Weyl points, and near the gap of the spin minority band. In (f) non-collinear antiferromagnetic state, the electronic band dispersion around the $\mathrm{L}$ point remains almost unchanged, comparing to that in ferromagnetic state.

determined to satisfy the following equation,

$$
n_{\mathrm{e}}=\int_{-\infty}^{\infty} d \epsilon \rho(\epsilon) f(\epsilon-\mu, T)
$$

Here, $\rho(\epsilon)$ is the density of states per unit cell, $k_{\mathrm{B}}$ is the Boltzman constant and $T$ is temperature. According to the above argument, we can determine the chemical potential $\mu$ using the Eq. 12.

\section{MAGNETIC ORDERING}

Next, we investigate the magnetic ordering with respect to the filling factor of dopants $\Delta n_{\mathrm{e}}$ and temperature $T$. In Fig. 3, the $\Delta n_{\mathrm{e}}-T$ dependence of (a) the $z$ component of magnetization $m^{z}=\sum_{\gamma}\left\langle m_{\gamma}^{z}\right\rangle$,

(b) the in-plane component of magnetization $m_{/ /}=$ $\sum_{\alpha} \sqrt{\left\langle m_{\alpha}^{x}\right\rangle^{2}+\left\langle m_{\alpha}^{y}\right\rangle^{2}} \quad(\alpha=\mathrm{A}, \mathrm{B}$, and $\mathrm{C}),(\mathrm{c})$ the $z$ component of the vector spin chirality 28] $(\boldsymbol{K})^{z}=\left(\boldsymbol{S}_{A} \times\right.$ $\left.\boldsymbol{S}_{B}+\boldsymbol{S}_{B} \times \boldsymbol{S}_{C}+\boldsymbol{S}_{C} \times \boldsymbol{S}_{A}\right)^{z}$ are shown. Additionally, in Fig. 3, the band structure and the density of states at (d) $\Delta n_{\mathrm{e}}=-1$, (e) $\Delta n_{\mathrm{e}}=0$, and (f) $\Delta n_{\mathrm{e}}=+1$ are shown. First, we study the FM ordering with the perpendicular anisotropy in undoped $\operatorname{CSS}\left(\Delta n_{\mathrm{e}}=0\right)$. Figures 3 (a) and 3 (b) show, at low temperature, $m^{z} \sim 0.9$ and $m_{/ /} \sim 0$ in undoped case $\left(\Delta n_{\mathrm{e}}=0\right)$, indicating FM ordering with the perpendicular anisotropy. The value $m^{z} \sim 0.9$ is consistent with results obtained by first-principles calculations 8 and experiment 8,29 . We find the critical temperature in undoped case being $T_{0}=$ $0.4 t_{1} / k_{\mathrm{B}}$. The band structure and the density of states in undoped case $\left(\Delta n_{\mathrm{e}}=0\right)$ obtained by the Hartree-Fock method are shown in Fig. 3(e). We set $k_{\mathrm{B}} T / t_{1}=0.01$. $E_{1} / t_{1}=0$ is set as the chemical potential $\mu$ obtained by Eq. 121. We do not depict the lower two bands because 
they are energetically apart from $\mu$. As the right panel of Fig. 3(e) shows, near $\mu$, the spin up band has a relatively small density of states corresponding to the Weyl points. Whereas the spin down band is close to the band gap. This describes the spin-polarized Weyl semimetalic state in undoped CSS.

Next, we show the suppression of the FM ordering in the hole-doped regime. Figure 3(a) shows that the FM transition temperature decreases when $\Delta n_{\mathrm{e}}<$ 0 . This suppression of FM ordering by hole-doping is consistent with experiment in $\mathrm{Co}_{3-x} \mathrm{Fe}_{x} \mathrm{Sn}_{2} \mathrm{~S}_{2}$ 3033 and first-principles calculations and experiment for $\mathrm{Co}_{3} \mathrm{In}_{x} \mathrm{Sn}_{2-x} \mathrm{~S}_{2}$ 27, 34. The non-magnetic band structure and the density of states in the hole-doped CSS when $\Delta n_{\mathrm{e}}=-1$ are shown in Fig. 3(d). In this situation, $\mu$ is close to the band gap, indicating a paramagnetic state with small carriers.

Then, we study the electron-doped regime. This situation could be realized experimentally in $\mathrm{Co}_{3-x} \mathrm{Ni}_{x} \mathrm{Sn}_{2} \mathrm{~S}_{2}$ [35, 36. As shown in Fig. 3(a), $m^{z}$ decreases as $\Delta n_{\mathrm{e}}$ increases from $\Delta n_{\mathrm{e}}=0$. As Fig. 3(c) shows, $z$ component of vector spin chirality becomes positive as $\Delta n_{\mathrm{e}}$ increases, while $m_{z}$ vanishes. Especially, when $\Delta n_{\mathrm{e}}=$ +1 , we find that the spin configuration becomes as $\boldsymbol{m}_{A}=m(1,0,0), \boldsymbol{m}_{B}=m(\cos (2 \pi / 3), \sin (2 \pi / 3), 0)$, $\boldsymbol{m}_{C}=m(\cos (4 \pi / 3), \sin (4 \pi / 3), 0)$, where $m \sim 0.5 \mu_{\mathrm{B}}$. These results conclude that the non-collinear AF ordering appears within the restricted order parameter space of our model. In Fig. 3(f), the electronic band structure and the density of states in the non-collinear AF state are shown. Around the L point, the band dispersion near $\mu$ remains almost unchanged from that in FM state [Fig. 3(e)]. In Fig. 3(c), the non-collinear AF ordering sustains up to $T / T_{0} \sim 2.3$. However, we note that the magnetic transition temperature is overestimated due to the use of Hartree-Fock method 37]. On the other hand, at low temperature the appearance of magnetic ordering is reliable.

\section{ORBITAL MAGNETIZATION IN ANTIFERROMAGNETIC STATE}

In the previous section, we showed that the noncollinear AF ordering appears in the electron-doped regime. Here, we discuss the orbital magnetization and the anomalous Hall conductivity, characterizing the noncollinear AF state. Considering a certain additional SOC, the orbital magnetization and the anomalous Hall conductivity become finite in the non-collinear AF state. We note that, by considering only the intralayer KaneMele SOC given by Eq. (3), both of these values vanish. As an additional interaction, we introduce the interlayer Kane-Mele type SOC due to the honeycomb structure.

$$
H_{\mathrm{KM}}^{z}=-\mathrm{i} t_{\mathrm{KM}}^{z} \sum_{\langle\langle i j\rangle\rangle \sigma \sigma^{\prime}} \boldsymbol{\eta}_{i j} \cdot d_{i \sigma}^{\dagger} \boldsymbol{\sigma}_{\sigma \sigma^{\prime}} d_{j \sigma^{\prime}}
$$
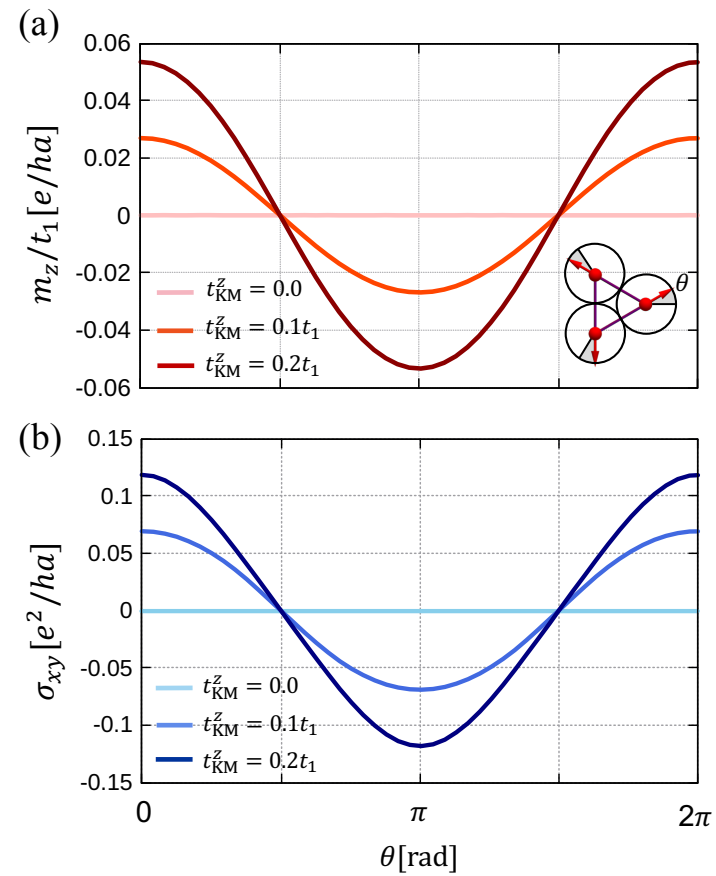

FIG. 4. (a) Orbital magnetizations and (b) anomalous Hall conductivity for $t_{\mathrm{KM}}^{z}=0.0,0.1 t_{1}$, and $0.2 t_{1}$, as a function of magnetization angle $\theta$ depicted in an inset of (a).

Here, $\boldsymbol{\eta}_{i j}$ are given by $\boldsymbol{\eta}_{C A}=\frac{\boldsymbol{a}_{1}}{2} \times \frac{\boldsymbol{a}_{3}}{2}, \boldsymbol{\eta}_{A B}=\frac{\boldsymbol{a}_{2}}{2} \times \frac{\boldsymbol{a}_{1}}{2}$, and $\boldsymbol{\eta}_{B C}=\frac{\boldsymbol{a}_{3}}{2} \times \frac{\boldsymbol{a}_{2}}{2}$. Although the magnetic ordering remains mostly unchanged by this additional SOC Eq. 113, this term makes the orbital magnetization and the $\mathrm{AHC}$ finite in non-collinear AF state.

We study the spin-moment angle dependences of the orbital magnetization. The orbital magnetization can be obtained by the formula $4,38,40$,

$$
\begin{aligned}
M_{\alpha}^{\mathrm{orb}}= & \frac{e}{2 \hbar} \sum_{\lambda} \int_{\mathrm{BZ}} \frac{d^{3} k}{(2 \pi)^{3}} f_{\lambda \boldsymbol{k}} \epsilon_{\alpha \beta \gamma} \\
& \times \operatorname{Im} \sum_{\lambda^{\prime} \neq \lambda} \frac{\left\langle\lambda, \boldsymbol{k}\left|\hbar v_{\beta}\right| \lambda^{\prime}, \boldsymbol{k}\right\rangle\left\langle\lambda^{\prime}, \boldsymbol{k}\left|\hbar v_{\gamma}\right| \lambda, \boldsymbol{k}\right\rangle}{\left(E_{\lambda^{\prime} \boldsymbol{k}}-E_{\lambda \boldsymbol{k}}\right)^{2}} \\
& \times\left(E_{\lambda^{\prime} \boldsymbol{k}}+E_{\lambda \boldsymbol{k}}-2 E_{\mathrm{F}}\right)
\end{aligned}
$$

Here, $v_{i}(i=x, y, z)$ is the velocity operator given by $v_{i}=\frac{1}{\hbar} \frac{\partial H(\boldsymbol{k})}{\partial k_{i}}$. The eigenstates $|\lambda, \boldsymbol{k}\rangle$ are obtained by diagonalizing $\mathcal{H}_{0}(\boldsymbol{k})+\mathcal{H}_{\text {exc }}$ with Eq. 13 . Figure 4 (a) shows $M_{z}^{\text {orb }}$ as a function of the angle of magnetic moment on kagome lattice for $t_{\mathrm{KM}}^{z}=0.0,0.1 t_{1}$ and $0.2 t_{1}$, . Each magnetic moment is rotated with an equivalent relative angle as shown in an inset of Fig. 4(a). $E_{\mathrm{F}}$ in Eq. (14) is obtained by $\Delta n_{\mathrm{e}}=+1$ condition and the magnetic order parameters on each site are obtained by HartreeFock method. $M_{z}^{\text {orb }}$ is finite and changes like a $\cos \theta$ function. We note that $M_{x}^{\text {orb }}=M_{y}^{\text {orb }}=0$. These results indicate that our model in the non-collinear AF 
state shows a finite orbital magnetization although the net magnetization vanishes. The direction of the spin moments can be changed by an external magnetic field as similarly discussed in Ref. [4. In the presence of an external magnetic field pointing the $z$ direction $B_{z}$, the orbital magnetization $M_{z}^{\text {orb }}$ couples as $-M_{z}^{\text {orb }} B_{z}$. When the external magnetic field points $+z$ direction, the spin angle $\theta=0$ is energetically favored. On the other hand, when the external magnetic field points $-z$ direction, the spin angle $\theta=\pi$ is energetically favored.

The change of the spin direction is related to the AHE. The intrinsic AHC $\sigma_{x y}$ can be calculated by the formula 41 given by,

$$
\begin{aligned}
\sigma_{x y}= & e^{2} \hbar \sum_{\lambda} \int_{\mathrm{BZ}} \frac{d^{3} k}{(2 \pi)^{3}} f_{\lambda \boldsymbol{k}} \\
& \times \operatorname{Im} \sum_{\lambda^{\prime} \neq \lambda} \frac{\left\langle\lambda, \boldsymbol{k}\left|v_{x}\right| \lambda^{\prime}, \boldsymbol{k}\right\rangle\left\langle\lambda^{\prime}, \boldsymbol{k}\left|v_{y}\right| \lambda, \boldsymbol{k}\right\rangle}{\left(E_{\lambda^{\prime} \boldsymbol{k}}-E_{\lambda \boldsymbol{k}}\right)^{2}} .
\end{aligned}
$$

As shown in Fig. 4(b), the angle dependence of the AHC is similar to that of the orbital magnetization in Fig. 4(a). Therefore, the sign of the AHC changes when the direction of spin moments is changed by an external magnetic field. Although the AHC in $\Delta n_{\mathrm{e}}=+1$ is smaller than that in ferromagnetic Weyl state $\left(\Delta n_{\mathrm{e}}=0\right)$ [23], the change of the direction of spin moments in non-collinear AF state might be detected by applying a uniform magnetic field.

\section{CONCLUSION}

In this paper, we investigated the magnetic ordering in an effective model of stacked-kagome lattice system CSS, based on the Hartree-Fock method. We showed the suppression of the perpendicular ferromagnetic ordering by hole doping. Non-collinear AF phase appears in electrondoped regimes and possesses finite orbital magnetization and the AHC by considering the interlayer SOC.

\section{ACKNOWLEDGEMENT}

We thank Y. Araki, K. Kobayashi, Y. Motome, A. Tsukazaki, J. Watanabe for valuable discussions. This work was supported by JSPS KAKENHI Grants No. 20H01830, JST CREST Grant No. JPMJCR18T2, and GP-Spin at Tohoku University.
[1] S. Nakatsuji, N. Kiyohara, and T. Higo, Nature 527, $212(2015)$.

[2] M.-T. Suzuki, T. Koretsune, M. Ochi, and R. Arita, Phys. Rev. B 95, 094406 (2017).

[3] J. Liu and L. Balents, Phys. Rev. Lett. 119, 087202 (2017).

[4] N. Ito and K. Nomura, J. Phys. Soc. Jpn 86, 063703 (2017).

[5] S.-S. Zhang, H. Ishizuka, H. Zhang, G. B. Halász, and C. D. Batista, Phys. Rev. B 101, 024420 (2020).

[6] L. Ye, M. Kang, J. Liu, F. von Cube, C. Wicker, T. Suzuki, C. Jozwiak, A. Bostwick, R. Rotenberg, D. Bell, L. Fu, R. Comin, and J. G. Checkelsky, Nature 555, 638 (2018).

[7] J.-X. Yin, S. S. Zhang, H. Li, K. Jiang, G. Chang, B. Zhang, B. Lian, C. Xiang, I. Belopolski, H. Zheng, T. Cochran, A.-Y. Xu, G. Bian, K. Liu, T.-R. Chang, H. Lin, Z.-Y. Lu, Z. Wang, S. Jia, W. Wang, and M. Hasan, Nature 562, 91 (2018).

[8] E. Liu, Y. Sun, N. Kumar, L. Muechler, A. Sun, L. Jiao, S.-Y. Yang, D. Liu, A. Liang, Q. Xu, Y. Sun, N. Kumar, L. Muechler, A. Sun, L. Jiao, S.-Y. Yang, D. Liu, A. Liang, Q. Xu, J. Kroder, V. Süß, H. Borrmann, C. Shekhar, C. Wang, Z. Xi, W. Wang, W. Schnelle, S. Wirth, Y. Chen, S. Goennenwein, and C. Felser, Nat. Phys. 14 (2018).

[9] Q. Xu, E. Liu, W. Shi, L. Muechler, J. Gayles, C. Felser, and Y. Sun, Phys. Rev. B 97, 235416 (2018).

[10] Q. Wang, Y. Xu, R. Lou, Z. Liu, M. Li, Y. Huang, D. Shen, H. Weng, S. Wang, and H. Lei, Nat.Commun. 9 (2018).
[11] D. Liu, A. Liang, E. Liu, Q. Xu, Y. Li, C. Chen, D. Pei, W. Shi, S. Mo, P. Dudin, T. Kim, C. Cacho, G. Li, Y. Sun, L. Yang, Z. K. Liu, S. Parkin, C. Felser, and Y. Chen, Science 365, 1282 (2019).

[12] M. Tanaka, Y. Fujishiro, M. Mogi, Y. Kaneko, T. Yokosawa, N. Kanazawa, S. Minami, T. Koretsune, R. Arita, S. Tarucha, M. Yamamoto, and Y. Tokura, Nano Lett. 20, 7476 (2020).

[13] X. Wan, A. M. Turner, A. Vishwanath, and S. Y. Savrasov, Phys. Rev. B 83, 205101 (2011).

[14] A. A. Burkov and L. Balents, Phys. Rev. Lett. 107, 127205 (2011).

[15] N. P. Armitage, E. J. Mele, and A. Vishwanath, Rev. Mod. Phys. 90, 015001 (2018).

[16] K. Barros, J. W. F. Venderbos, G.-W. Chern, and C. D. Batista, Phys. Rev. B 90, 245119 (2014).

[17] J. Ikeda, K. Fujiwara, J. Shiogai, T. Seki, K. Nomura, K. Takanashi, and A. Tsukazaki, Commun. Mater. 2, 1 (2021).

[18] J. Shiogai, J. Ikeda, K. Fujiwara, T. Seki, K. Takanashi, and A. Tsukazaki, Phys. Rev. Mater. 5, 024403 (2021).

[19] Z. Guguchia, J. Verezhak, D. Gawryluk, S. Tsirkin, J.X. Yin, I. Belopolski, H. Zhou, G. Simutis, S.-S. Zhang, T. Cochran, G. Chang, E. Pomjakushina, Z. Keller, L. Skrzeczkowska, Q. Wang, R. Lei, H.C. Khasanov, A. Amato, T. Jia, A. Neupert, H. Luetkens, and M. Hasan, Nat. Commun. 11 (2020).

[20] Z. Guguchia, H. Zhou, C. Wang, J.-X. Yin, C. Mielke, S. Tsirkin, I. Belopolski, S.-S. Zhang, T. Cochran, T. Neupert, R. Khasanov, A. Amato, M. Jia, S. Hasan, and H. Luetkens, npj Quantum Mater. 6, 1 (2021). 
[21] Q. Zhang, S. Okamoto, G. D. Samolyuk, M. B. Stone, A. I. Kolesnikov, R. Xue, J. Yan, M. A. McGuire, D. Mandrus, and D. A. Tennant, Phys. Rev. Lett. 127, 117201 (2021).

[22] K. Yoshida, in Theory of Magnetism (Springer Series in Solid-State Sciences, 1996).

[23] A. Ozawa and K. Nomura, J. Phys. Soc. Jpn. 88, 123703 (2019).

[24] C. L. Kane and E. J. Mele, Phys. Rev. Lett. 95, 226801 (2005).

[25] H.-M. Guo and M. Franz, Phys. Rev. B 80, 113102 (2009).

[26] W. Luo, Y. Nakamura, J. Park, and M. Yoon, npj Comput. Mater. 7 (2021).

[27] Y. Yanagi, J. Ikeda, K. Fujiwara, K. Nomura, A. Tsukazaki, and M.-T. Suzuki, Phys. Rev. B 103, 205112 (2021).

[28] D. Grohol, K. Matan, J.-H. Cho, S.-H. Lee, J. W. Lynn, D. G. Nocera, and Y. S. Lee, Nat. Mater. 4, 323 (2005).

[29] M. A. Kassem, Y. Tabata, T. Waki, and H. Nakamura, Phys. Rev. B 96, 014429 (2017).

[30] R. Weihrich and I. Anusca, Z. Anorg. Allg. Chem. 632, 1531 (2006).
[31] M. A. Kassem, Y. Tabata, T. Waki, and H. Nakamura, J. Solid State Chem. 233, 8 (2016).

[32] H. Zhou, G. Chang, G. Wang, X. Gui, X. Xu, J.-X. Yin, Z. Guguchia, S. S. Zhang, T.-R. Chang, H. Lin, W. Xie, M. Z. Hasan, and S. Jia, Phys. Rev. B 101, 125121 (2020).

[33] J. Shen, Q. Zeng, S. Zhang, H. Sun, Q. Yao, X. Xi, W. Wang, G. Wu, B. Shen, Q. Liu, and L. E., Adv. Funct. Mater. 30, 2000830 (2020).

[34] M. A. Kassem, Y. Tabata, T. Waki, and H. Nakamura, J. Cryst. Growth 426, 208 (2015).

[35] T. Kubodera, H. Okabe, Y. Kamihara, and M. Matoba, Physica B 378, 1142 (2006).

[36] G. S. Thakur, P. Vir, S. Guin, C. Shekhar, R. Weihrich, Y. Sun, N. Kumar, and C. Felser, Chem. Mater. 32, $1612(2020)$.

[37] X. Dai, K. Haule, and G. Kotliar, Phys. Rev. B 72, 045111 (2005).

[38] D. Xiao, M.-C. Chang, and Q. Niu, Rev. Mod. Phys. 82, 1959 (2010).

[39] T. Thonhauser, D. Ceresoli, D. Vanderbilt, and R. Resta, Phys. Rev. Lett. 95, 137205 (2005).

[40] Y. Ominato, S. Tatsumi, and K. Nomura, Phys. Rev. B 99, 085205 (2019).

[41] N. Nagaosa, J. Sinova, S. Onoda, A. H. MacDonald, and N. P. Ong, Rev. Mod. Phys. 82, 1539 (2010). 